\title{
latrogenic infection of a colonic cystic lymphangioma following cold-forceps biopsy
}

An incidental 4-cm, cecal, subepithelial compression with a smooth mucosa was found during colonoscopy in a 63-year-old man ( $\bullet$ Fig. 1). A computed tomography (CT) scan demonstrated a $4.4 \times 2.7-\mathrm{cm}$, cystic cecal mass ( $\bullet$ Fig. 2 ). A 12-MHz, through-the-scope, endoscopic ultrasound (EUS) miniprobe demonstrated an anechoic, multiseptated, submucosal cecal cyst with intact muscularis propria ( $\bullet$ Fig.3), consistent with a colonic cystic lymphangioma (CCL). To document the benign overlying mucosa, a standard, cold-forceps pinch biopsy (Boston Scientific Corp., Natick, Massachusetts, USA) was performed, which resulted in drainage of a small amount of whitish fluid.

Sixteen hours later, the patient developed abdominal pain, chills and a low grade fever $\left(100^{\circ} \mathrm{F}\right)$. A repeat CT scan demonstrated an increase in the size of the cecal cyst with air pockets and pericecal stranding suggestive of infection ( Fig.4). Intravenous broad-spectrum antibiotics were given for the first 24 hours, followed by oral antibiotics, which were continued for a total of 14 days. The biopsy subsequently revealed colonic mucosa with markedly dilated lymphatic channels confirming CCL ( $\bullet$ Fig.5). Successive patient follow-up ensured the clinical resolution of all symptoms.

Lymphangiomas are rare benign lymphatic tissue malformations. Endoscopically, CCLs appear as a subepithelial compression. Demonstration of an anechoic, multiseptated, submucosal cyst by EUS is conceivably sufficient for diagnosis $[1,2]$. Because CCLs are benign, asymptomatic patients can be managed conservatively by observation [1]. Definitive therapy includes segmental or radical resection for larger lymphangiomas $(>2.5 \mathrm{~cm})[3,4]$. To our knowledge, there are no published cases of iatrogenic infection of CCLs. In this case, a forceps biopsy was obtained primarily for histological confirmation of the normal overlying mucosa. The resultant unanticipated infection of the CCL indicates the risks involved with such interventions.

In conclusion, EUS is essential for the noninvasive diagnosis of CCLs. An endoscopic biopsy of the overlying normal-appearing

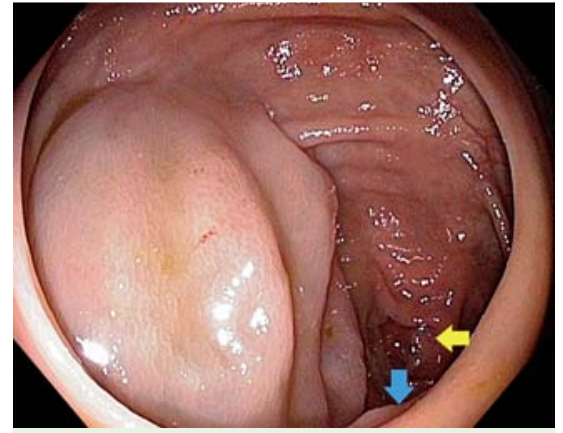

Fig. 1 Colonoscopic view of a 4-cm, subepithelial compression in the cecum with overlying smooth mucosa (the yellow arrow indicates the appendiceal orifice; the blue arrow indicates the ileocecal valve).

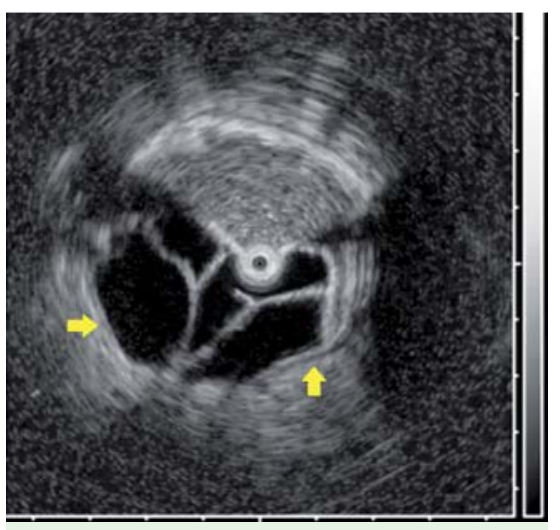

Fig. 3 Endoscopic ultrasound (EUS) with a 12-MHz frequency, through-the-scope, ultrasound miniprobe demonstrating a $4.3 \times 2.9$ $\mathrm{cm}$, anechoic, multiseptated, submucosal cystic lesion, consistent with a cystic lymphangioma. Intact muscularis propria (yellow arrows) is visible underneath the lesion.

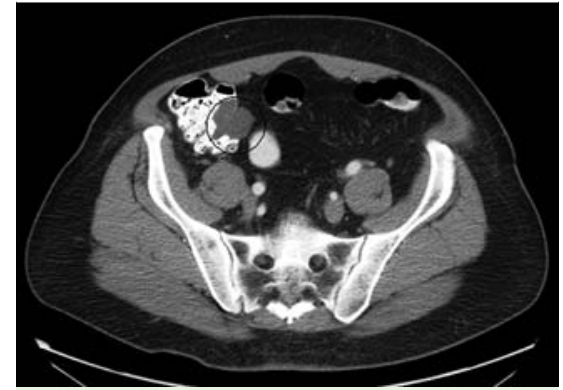

Fig. 2 Abdominopelvic computed tomography $(C T)$ scan demonstrating a $4.4 \times 2.7-\mathrm{cm}$, cystic, cecal wall mass (circled), which is separate from the appendix.

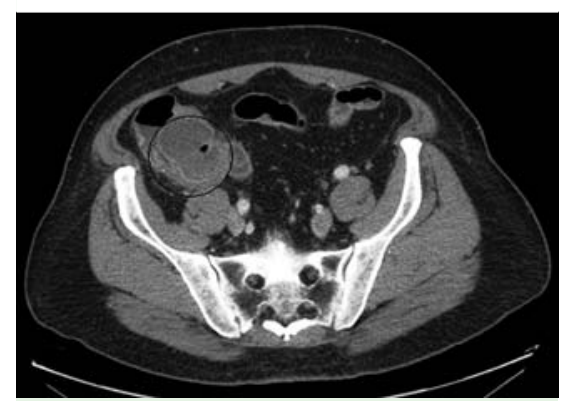

Fig. 4 Repeat abdominopelvic computed tomography (CT) scan 16 hours later showing the increased size of the cecal cyst (circled), which is now $6.3 \times 3.4 \mathrm{~cm}$, with an air-fluid level and adjacent stranding visible, raising the possibility of an infection.

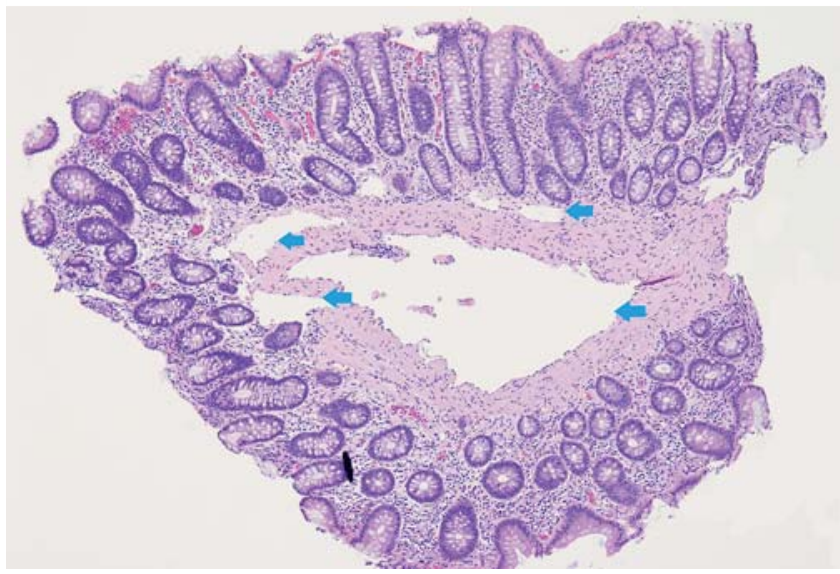

Fig. 5 Histological appearance of the biopsy specimen showing dilated lymphatic channels in the lamina propria and muscularis mucosae (blue arrows) with overlying unremarkable mucosa (hematoxylin and eosin [H\&E] stain; magnification $\times 40$ ). 
mucosa may lead to an infectious complication. Forceps biopsy may not be necessary if the EUS image is typical for a benign CCL. If it is deemed clinically necessary to perform such a biopsy, we would suggest, based on our experience, the use of prophylactic antibiotics to prevent an infectious complication.

Endoscopy_UCTN_Code_CPL_1AJ_2AB

\section{Competing interests: None}

\section{S. G. Krishna', J. R. Stroehlein ${ }^{1}$,} W. C. Foo ${ }^{2}$, R. Suzuki ${ }^{1}$, S. R. Reddy ${ }^{1}$, M. S. Bhutani ${ }^{1}$

${ }^{1}$ Department of Gastroenterology, Hepatology and Nutrition, University of Texas MD Anderson Cancer Center, Houston, Texas, USA

${ }^{2}$ Department of Department of Pathology and Laboratory Medicine, University of Texas MD Anderson Cancer Center, Houston, Texas, USA

\section{References}

1 Irisawa A, Bhutani MS. Cystic lymphangioma of the colon: endosonographic diagnosis with through-the-scope catheter miniprobe and determination of further management. Report of a case. Dis Colon Rectum 2001; 44: $1040-1042$

2 Gottlieb K, Elkharwily A. Endoscopic ultrasound evaluation of a cystic lymphangioma of the colon. J Ultrasound Med 2007; 26: $1803-1804$

3 Huguet KL, Metzger PP, Menke DM. Colorectal lymphangioma. Am Surg 2007; 73: 414416

4 Matsuda T, Matsutani T, Tsuchiya $Y$ et al. A clinical evaluation of lymphangioma of the large intestine: a case presentation of lymphangioma of the descending colon and a review of 279 Japanese cases. J Nihon Med Sch 2001; 68: $262-265$
Bibliography

DoI http://dx.doi.org/ 10.1055/s-0031-1291568

Endoscopy 2012; 44: E104-E105

(C) Georg Thieme Verlag KG

Stuttgart · New York

ISSN 0013-726X

\section{Corresponding author}

\section{S. Bhutani, MD}

Department of Gastroenterology, Hepatology and Nutrition

University of Texas MD Anderson Cancer Center 1400 Hermann Pressler Dr Houston, TX 77030-4008 USA

Fax: +1-713-563-4408

Manoop.Bhutani@mdanderson.org 